\title{
Vaccination with toxofilin DNA in combination with an alum- monophosphoryl lipid A mixed adjuvant induces significant protective immunity against Toxoplasma gondii
}

Pengxia Song', Shenyi He ${ }^{1 *}$, Aihua Zhou ${ }^{3}$, Gang Lv', Jingjing Guo', Jian Zhou², Yali Han', Huaiyu Zhou', Zhen $\mathrm{HaO}^{1}$ and Hua Cong ${ }^{1}$

\begin{abstract}
Background: A widely prevalent disease, toxoplasmosis poses serious health threats to both humans and animals; therefore, development of an ideal DNA vaccine against Toxoplasma gondii is needed eagerly. The purpose of the present study is to assess the protective efficacy of a DNA vaccine encoding the $T$. gondii toxofilin gene (pEGFP-toxofilin). In addition, toxofilin DNA vaccine combined with the individual adjuvants, alum or monophosphoryl lipid A (MPLA), or a mixture of alum-MPLA adjuvant were screened for their ability to enhance antibody responses.

Methods: Using bioinformatics, we analyzed the gene and amino acid sequences of the toxofilin protein, recognizing and identifying several potential linear B and T helper (Th)-1 cell epitopes. BALB/c mice were immunized three times with either toxofilin DNA vaccine alone or in combination with the adjuvants such as alum, MPLA or an alum-MPLA mixture. The systemic immune response was evaluated by cytokine, the percentage of CD4 $(+)$ and CD8 (+) T cells and specific antibody measurement. Two weeks after the last immunization, protective efficacy was evaluated by challenging intraperitoneally with $1 \times 10^{4}$ tachyzoites of $T$. gondii or intragastrically with 20 cysts of T. gondii PRU strain.

Results: All experimentally immunized mice developed strong humoral and cellular immune responses compared with the control groups. Moreover, by comparison with the non-adjuvant toxofilin DNA vaccine group, adding alum adjuvant to toxofilin DNA vaccine resulted in an increase in humoral response and a skewed Th2 response. However, the MPLA adjuvant with toxofilin DNA vaccine induced significantly enhanced humoral and Th1-biased immune responses. Importantly, the co-administration of alum-MPLA adjuvant in combination with the toxofilin DNA vaccine shifted the Th2 immune response to a Th1 response compared with the alum-toxofilin group, and elicited the strongest humoral and Th1 responses among all the groups. At the same time, a longer survival time and less cyst amounts against T. gondii infection were also observed in the alum-MPLA-toxofilin group in comparison with single or no adjuvant groups.

(Continued on next page)
\end{abstract}

\footnotetext{
* Correspondence: shenyi.he@hotmail.com

'Department of Parasitology, Shandong University School of Medicine, Jinan,

Shandong Province 250012, People's Republic of China

Full list of author information is available at the end of the article
} 
(Continued from previous page)

Conclusions: Toxoplasma gondii toxofilin is a promising vaccine candidate that warrants further development. Co-administration of the alum-MPLA adjuvant mixture with DNA vaccine could effectively enhance immunogenicity and strongly skew the cellular immune response towards a Th1 phenotype.

Keywords: Toxoplasma gondii, Toxofilin, Bioinformatics, DNA vaccine, Adjuvant

\section{Background}

Toxoplasma gondii is an obligate intracellular protozoan parasite [1]. It has a complex life cycle, infecting a broad host range of mammals and birds as intermediate hosts but with felids as the only definitive hosts [2]. The T.gondii has a wide range of distribution and high infection rates in many parts of the world and a third of population is infected [3, 4]. Although infection with $T$. gondii may be asymptomatic in immunocompetent individuals, severe disease can develop in immunocompromised patients or fetuses of seronegative women undergoing primary infection during pregnancy $[4,5]$.

The primary strategy for the treatment of toxoplasmosis is chemical drugs; however, such drugs have many drawbacks, including poor tolerance, side effects, and the development of drug resistance and are ineffective for the treatment of $T$. gondii tissue cysts in warmblooded animals. Besides, they can't prevent reinfection [6]. Therefore, the development of an effective and safe vaccine against toxoplasmosis is of great importance to prevent the continued spread of this disease. In the past 15 years, significant progresses have been made in the development of vaccine [7]. DNA vaccinations against toxoplasmosis are well known to induce Th1 immune response [8]. Several single-antigen vaccines such as ROP8, TgCyP, and GRA6, have been successful in facilitating survival following a lethal dose of infection challenge, with a range of survival rates from 37.5 to $50.0 \%$ [9-11]. However, the development of a higher efficacy vaccine remains a vital challenge in light of the persistent nature of chronic toxoplasmosis.

Toxofilin, which was discovered in the rhoptry proteome, was originally based on its role in binding and the regulation of host actin dynamics. Recently, toxofilin was crystallized in complex with mammalian actin, establishing the interaction between the parasite and host protein [12]. Toxofilin is an internal protein. During the parasites invade into the host cell, the protein was secreted to host cell to facilitate vacuole folding and tachytozoites invasion. In addition, antitoxofilin or anti-HA antibodies was detected in infected host cells by fluorescence microscopy imaging [13]. However, there is thus far no evidence as to whether toxofilin DNA vaccine can induce significant humoral and cellular responses in mice and the protective efficacy of toxofilin DNA as a vaccine against $T$. gondii remains unclear.

A good vaccine also depends on the choice of adjuvants which play a key factor in dictating the effectiveness of the immune response [14, 15]. Aluminum salts (alum) are the most widely used of adjuvants in vaccine development. However, alum which is used as an adjuvant has several limitations. Obviously, alumbased adjuvants tend to $\mathrm{T}$ helper (Th) 2 immunity and are incompatible with small peptide antigens [16]. With the aim of developing a transmission-blocking T.gondii vaccine, the application of compound adjuvant has leaded the vaccine development to a new era, resulting in a stronger immune response through a synergistic effect. This strategy incorporating a combination of adjuvants (monophosphoryl lipid A (MPLA) and alum) is proven to be effective $[17,18]$. MPLA as an adjuvant system have additive effects, especially intracellular processing of Th1 antigens by the major histocompatibility complex. At the same time, MPLA can enhance antigenspecific induction of antibodies to a variety of antigens $[19,20]$.

To our knowledge, no report has described a toxofilinbased vaccination regimen against $T$. gondii. In this study, the linear B- and Th-cell epitopes of toxofilin protein were analyzed using bioinformatics. Furthermore, through analysis of the generated peptide segment tables, we constructed a eukaryotic plasmid pEGFPtoxofilin and investigated the overall survival, cysts burden and levels of humoral and cellular immune responses elicited by toxofilin DNA vaccine alone or in combination with one and more adjuvants following intraperitoneal (i.p.) or intragastrically challenge or in $\mathrm{BALB} / \mathrm{c}$ mice.

\section{Methods}

\section{Bioinformatic analysis}

The gene and amino acid sequences of toxofilin were identified from GenBank (http://www.ncbi.nlm.nih.gov/ genbank), the primary characteristics of the gene encoding protein were analyzed by ProtParam (http://web.expasy.org/protparam/), the primary amino acid sequences of toxofilin homologs were downloaded from the National 
Center for Biotechnology Information, and the multiple sequence alignment was generated using the Clustal W method in DNASTAR MegAlign software.

\section{Bioinformatic tools for predicting potential B-cell-binding epitopes}

$\mathrm{B}$-cell epitopes are recognized in their native structure by $\mathrm{B}$-cell receptors or antibodies. Continuous B cell epitope prediction is mainly according to the amino acid properties. However the discontinuous B cell epitope prediction requires $3 \mathrm{D}$ structure of the antigen [21-23]. In this study, the complete amino acid sequence and the secondary structure of toxofilin were analyzed using DNASTAR [24]. A higher score of toxofilin corresponded to a higher probability of the residue being involved in the epitope, using DNAMA software. The SOPMA and I-TASSER online services were used to construct the three-dimensional (3D) structures of the toxofilin epitopes [25].

\section{Identification of the Th-cell epitopes}

The Immune Epitope Database online service (http:// tools.immuneepitope.org/analyze/html/mhc_II_binding. html) was used to predict MHC II molecules of toxofilin. It is important to note, however, that such binding to $\mathrm{MHC}$ is necessary but not sufficient for recognition by $\mathrm{T}$ cells [26].

\section{Cell culture}

HEK239T cells stored at $-80{ }^{\circ} \mathrm{C}$ were thawed for $1 \mathrm{~min}$ at $37{ }^{\circ} \mathrm{C}$ followed by culture in Dulbecco's modified Eagle's medium (DMEM; GIBCO) with 10\% fetal bovine serum, $100 \mathrm{mg} / \mathrm{mL}$ streptomycin, and $100 \mathrm{IU} / \mathrm{mL}$ penicillin at $37{ }^{\circ} \mathrm{C}$ in $5 \% \mathrm{CO}_{2}$.

Macrophages were cultured at $37{ }^{\circ} \mathrm{C}$ in DMEM with $4 \mathrm{mM} \mathrm{L}$-glutamine (Dutscher) supplemented with $10 \%$ heat-inactivated fetal calf serum (Dutscher) and Hepes $10 \mathrm{mM}$ (Dutscher), in a $5 \% \mathrm{CO}_{2}$ atmosphere.

\section{Mice and parasites}

Six- to eight-week-old female BALB/c mice were purchased from Shandong University Laboratory Animal Centre (Jinan, China). All animal experiments were approved with the Animal Ethics Committee of Shandong University under Contract 2011-0015.

We used two strains of T. gondii (RH and PRU) in this study. The low virulent strain (PRU strain) of T. gondii was kept in our laboratory by passage of cysts in Kunming mice. The tachyzoites of the RH strain of $T$. gondii, which was preserved at $-80{ }^{\circ} \mathrm{C}$, were obtained by passage on macrophages. About $1 \times 10^{4}$ tachyzoites of intermediate virulence were collected from one $225-\mathrm{cm}^{2}$ culture flask, corresponding to $1 \mu \mathrm{g}$ of TE. Tachyzoites of highly virulent $T$. gondii were collected to challenge mice.

\section{The construction of the expression plasmids}

The T. gondii toxofilin gene coding sequence [GenBank: AJ132777.2] was amplified by PCR from genomic parasite DNA using the following specific primer pair. Toxofilin for eukaryotic expression plasmid construction: forward primer: 5 '-CGGGGTACCATGGCGCAAT ACAAGTCACG-3'(Kpn I), reverse primer: 5'-CG GG ATCCTTACGACGAGGGCATAGCG-3'(BamH I). Toxofilin for prokaryotic expr -ession plasmid construction: forward primer: 5'-CGGGGTACCATGGCGCAA TACAA GTCACG-3'(Kpn I), reverse primer: 5'-CGGCGGCCGCT TACGACGA GGGCATAGCG-3" (Not I) (recognition sites for Kpn I, BamH I and Not I, respectively, are underlined). The amplified PCR products were cloned into the eukaryotic expression vector pEGFP-C1 (TransGen, China) and the prokaryotic expression plasmid pET-30a $(+)$ to formed a recombinant plasmid, pEGFP-toxofilin and pET30-toxofilin.

pEGFP-toxofilin plasmids expression in vitro $p$ EGFP-toxofilin plasmids were transformed into Escherichia coli DH5 by the way of hot shot $\left(42{ }^{\circ} \mathrm{C}\right.$.). Expression of two genes in transformed DH5 $\alpha$ cells was screened on LK solid medium, followed by choosing a single colony to cultivate for harvest of recombinant plasmids. All the recombinant plasmids were extracted by an endotoxin-free plasmid purification kit (Tiangen, China) and stored at $-20{ }^{\circ} \mathrm{C}$ until use. The plasmids concentrations were determined by A260/A280 measurement.

HEK239T cells grown in 6-well plates were respectively transfected with recombinant eukaryotic plasmid (pEGFP-toxofilin) or an empty plasmid pEGFP-C1 with the assistance of LipofectamineTM 2000 reagent (Invitrogen, USA) in accordance with the manufacturer's instructions. Protein expression in vitro was observed by specific green fluorescence on HEK293T cells after incubation for $24 \mathrm{~h}$ at $37{ }^{\circ} \mathrm{C}$ in a $5 \% \mathrm{CO}_{2}$ incubator. At $36 \mathrm{~h}$ post-transfection, the cells were rinsed twice with $\mathrm{PBS}$, handled with lysis buffer (Sigma, USA), and centrifuged at $16,000 \times \mathrm{g}$ for $30 \mathrm{~min}$. The lysates of transfected cells were analyzed by western blotting.

Expression and purification of pET30- toxofilin in bacteria Escherichia coli $\mathrm{DH} 5 \alpha$ strain cells were transformed by recombinant pET30-toxofilin and grown in Luria Bertani (LB) at $37^{\circ} \mathrm{C}$ until the cell density reached an absorbance of 0.6 (at $\lambda=600 \mathrm{~nm}$ ). Synthesis of recombinant pET30-toxofilin protein was induced with $1 \mathrm{mM}$ isopropyl- $\beta$-D-thiogalactoside (IPTG) for $6 \mathrm{~h}$ and $8 \mathrm{~h}$ at 
$25{ }^{\circ} \mathrm{C}$ and the growth for the next $3 \mathrm{~h}$ at $37{ }^{\circ} \mathrm{C}$. The cells were lysed and the protein was purified. The endotoxin was removed with the ToxinEraser ${ }^{\mathrm{rm}}$ Endotoxin Removal Kit and measured by the Chromogenic End-point Endotoxin Assay Kit (Chinese Horseshoe Crab Reagent Manufactory, Xiamen, China). Expression of the pET30toxofilin protein was analyzed by western blotting and kept at $-80{ }^{\circ} \mathrm{C}$ for further use.

\section{Immunization and challenge}

The immunization experiments that pEGFP-toxofilin DNA was used as the vaccine were performed in 6- to 8-week-old female BALB/c mice, which were randomly divided into eight groups ( $n=25 /$ group). Mice were injected intramuscularly three times at fortnightly intervals with the following four experimental immunizations: non-adjuvanted toxofilin (50 $\mu \mathrm{g}$ toxofilin DNA $+100 \mu \mathrm{L} \mathrm{PBS} /$ mouse); alum-toxofilin (50 $\mu \mathrm{g}$ toxofilin $\mathrm{DNA}+50 \mu \mathrm{g}$ alum $+50 \mu \mathrm{L}$ PBS/mouse); MPLA-toxofilin (50 $\mu \mathrm{g}$ toxofilin DNA + 50 $\mu \mathrm{g}$ MPLA $+50 \mu \mathrm{L}$ PBS/ mouse); alum-MPLA-toxofilin (50 $\mu \mathrm{g}$ toxofilin DNA + $50 \mu \mathrm{g}$ MPLA + $50 \mu \mathrm{L}$ PBS/mouse); and 4 control groups, with mice receiving $150 \mu \mathrm{L}$ PBS, $150 \mu \mathrm{g}$ alum, $150 \mu \mathrm{g}$ MPLA, $150 \mu \mathrm{g}$ alum-MPLA. The blood of mice in each group was collected at $0,2,4$, and 6 weeks by orbital blood and sera were stored at $-20{ }^{\circ} \mathrm{C}$ until used for subsequent analysis. Two weeks after the final inoculation, 12 mice in each group were challenged i.p. with $1 \times 10^{4}$ T. gondii RH strain tachyzoites, and the remaining mice in all groups were inoculated with 20 cysts of the attenuated virulent PRU strain orally. The individual survival time injected with RH strain along with the overall percentage survival were recorded. The dead mice infected $\mathrm{RH}$ strain were treated by high pressure sterilization and sent to the animal experiment center for further processing. The brain cysts were determined one month later after the challenge infection with 20 cysts of the attenuated virulent PRU strain orally for a one time. Each brain was homogenized in $2 \mathrm{ml} \mathrm{PBS}$, and the mean number of cysts per brain was calculated. This analysis was performed in three independent experiments.

\section{Determination of antibodies by ELISA}

Anti-T. gondii IgG, IgG1, and IgG2a antibodies were detected using enzyme-linked immunosorbent assay (ELISA) in accordance with the manufacturer's instructions (Southern Biotech Co., Ltd, Birmingham, USA). Briefly, microtiter 96-well plates were coated overnight at $4{ }^{\circ} \mathrm{C}$ with $\mathrm{pET} 30$-toxofilin protein antigen (diluted in $0.1 \mathrm{M}$ carbonate buffer $(\mathrm{pH}$ 9.5) for optimal binding. The plates were washed three times with PBS containing 0.05\% Tween20 (PBST) and then blocked with $1 \%$ bovine serum albumin for $1 \mathrm{~h}$ at $37{ }^{\circ} \mathrm{C}$ with gentle shaking. After three washes with
PBST, the plates were incubated with mouse sera diluted 1:100 in PBS for $1 \mathrm{~h}$, followed by incubation with goat anti-mouse IgG, IgG1, or IgG2a secondary antibodies for $1 \mathrm{~h}$ to detect the target antibody and isotype control, respectively. Finally, the immune enzymatic reaction complexes were visualized by incubating with ortho-phenylenediamine (Sigma, USA) and $0.15 \% \mathrm{H}_{2} \mathrm{O}_{2}$ for $30 \mathrm{~min}$. Reactions were stopped by adding $2 \mathrm{M} \mathrm{H}_{2} \mathrm{SO}_{4}$, and the results were read at OD450 $\mathrm{nm}$ using an ELISA plate reader. An average of three independent ELISAs for each serum sample was recorded.

\section{Cytokine assays}

Cytokine levels were determined as previously described [16]. Three mice per group were euthanized and their splenocytes aseptically harvested after two weeks after the last immunization, Cells were dispensed into 96-well plates at a density of $5 \times 10^{5}$ cells at $37^{\circ} \mathrm{C}$ with $95 \%$ relative humidity in $5 \% \mathrm{CO}_{2}$. Cell-free supernatants were harvested and assayed for interleukin (IL)4, IL-10, and IFN- $\gamma$ at $24 \mathrm{~h}, 72 \mathrm{~h}$, and $96 \mathrm{~h}$, respectively, using a commercial ELISA kit. All measurements were run in triplicate.

\section{Identification of CD4+ and CD8+ T cells by flow cytometry}

By flow cytomety analysis, the percentage of $\mathrm{CD} 4+$ and $\mathrm{CD} 8+\mathrm{T}$ cell in spleen were determined. The cell concentration was adjusted to $1 \times 10^{6}$ cells $/ \mathrm{ml}$ in PBS containing $2 \%$ FBS. After incubation with FITCconjugated anti-mouse CD4+ monoclonal antibody $(\mathrm{mAb}), \mathrm{PE}-$ conjugated anti-mouse $\mathrm{CD} 3+\mathrm{mAb}$ and Cy5.5-conjugated anti-mouse CD8+ $\mathrm{mAb}$ (eBioscience) at $4{ }^{\circ} \mathrm{C}$ for $30 \mathrm{~min}$ in the dark. The cultures were washed by $2 \mathrm{~mL}$ PBS and the suspensions were analyzed using SYSTEM II software (Coulter) through FACScan flow cytometer (BD Biosciences, San Jose, CA, USA).

\section{Statistical analyses}

All statistical analyses and graph plotting were performed by SPSS13.0 Data Editor and the differences in the data (antibody responses, lymphoproliferation assays, cytokine production and brain cyst burden) between all the groups were compared by one-way analysis of variance (ANOVA). Tukey's studentized range test was used for post-test comparisons. Survival rates for the experimental and control mice were compared using the Kaplan-Meier method and compared with the log-rank test. The differences were considered significantly if $P<0.05$. 


\section{Results}

Bioinformatic analysis

The toxofilin protein of $T$. gondii (Tg-toxofilin) is a 245 amino acid protein with a molecular weight of $27.1317 \mathrm{kDa}$. Its physical and chemical properties include a theoretical PI of 9.57, an instability index of 60.41, an aliphatic index of 84.98 , and a grand average of hydropathicity of -0.63 . For four different $T$. gondii strains, the protein sequence alignment is shown in Fig. 1. and share 96.53\% similarity, with toxofilin of the $T$. gondii $\mathrm{RH}$ strain sharing $100 \%, 91.84 \%$, and $94.29 \%$ sequence identity with GT1, ME49, and VANDPR strains, respectively.

\section{Secondary structure and 3D modeling}

B-cell epitopes are the portions of antigens that are recognized by the variable regions of antibodies. Accurately predicting epitopes is of great help for designing immunogenic peptides and new vaccines. In the present study, the DNASTAR software was used to predict antigenic index and surface probability, as shown in Fig. 2. Based on this analysis, the peptides with good hydrophilicity, high accessibility, high flexibility, and strong antigenicity were chosen as antigen epitopes. To further confirm these results, the DNAMAN software was used to analyze these sequences and nine potential epitopes were chosen with the highest antigen index scores, as shown in Table 1.

A potentially antigenic region of a protein increases the epitope prediction and promote a better understanding the 3D structure, contributing to the elucidation of the relationship between structure and function. The SOPMA and I-TASSER online services were used to predict the 3D structures of toxofilin alone and in complex with mammalian actin (Fig. 3 A1 and A2).

\section{Identification of the Th-cell epitopes}

The Th-cell epitopes identified on toxofilin by bioinformatic analyses reportedly have the ability to bind strongly to MHC class II molecules (Table 2). It is known that the binding force increases can influence Th-cell differentiation and promote more cells to differentiate into Th-1 cells [15]. Therefore, we speculate that toxofilin has the potential to induce Th-1 cell-mediated immune responses.

Identification of restructuring eukaryotic, prokaryotic and pro expression plasmids

Forty-eight hours after transfection of HEK cells, the presence of pEGFP-toxofilin or pEGFP-C1 was determined by fluorescence microscopy. As shown in Fig. 4(A1) and (A2), green fluorescence was observed in HEK293T cells transfected with pEGFP-toxofilin and pEGFP-C1, whereas there was no signal in the untransfected cells Fig. 4 (A3). Western blot analysis of these proteins was shown in Fig. 4B; a specific protein band (about $27 \mathrm{kDa}$ ) was recognized in cells transfected with pEGFP-toxofilin by incubation with a $T$. gondii antibody (lanes 2), whereas the negative controls transfected with empty pEGFP vector showed no specific bands upon incubation with a $T$. gondii antibodies (lane 1). The purified recombinant pET30toxofilin vector was used to produce toxofilin protein antigen. Western blot technique by using a $\mathrm{T}$. gondii

\begin{tabular}{|c|c|c|c|c|}
\hline T. gondi i & RH. seq & 1 & MAQYKSRPLAAFLLLITVGSLLTASESVQLSEGMKRLSMRGRSPSPKRGRFESGDEGTSTMSPSVAARQQELGLLRPEER & 80 \\
\hline T. gondi i & VANDPR. seq & 1 & MAQYKSRPLAAFLLLI TVGSLLTASESVQLSEGMKRLSMRGRSPSPKRGRFESGDEGTSTMSPSVAARQQELGLLRPEER & 80 \\
\hline T. gondi i & ME49. seq & 1 & MAQYKSRPLAAVLLLI TVGSLLTASESVQLSEGMKRLSMRGRSPSPKTGRFESGDEGTSTMSPSVAARQQELGLLRPEER & 80 \\
\hline T. gondi i & GT1. seq & 1 & MAOYKSRPLAAFLLLITVGSLLTASESVQLSEGMKRLSMRGRSPSPKRGRFESGDEGTSTMSPSVAARQQELGLLRPEER & 80 \\
\hline T. gondi i & RH. seq & 81 & LIAGQAKAAALQTVHQLGAVALTPEQAKAALLDE ILRATQNLDLRKYENLNTEQQKAYEQVQRDLSQLSPETKALLIENQ & 160 \\
\hline T. gondi i & VANDPR. seq & 81 & LIAGQAKAAALOTVHQLGAVVLTPEOAKAALLDEVLRATQNLDLRKYENLNTEQQKAYEOVQKDLSOLSPETKALLIENO & 160 \\
\hline T. gondi i & ME49. seq & 81 & LIAGQAKAAALOTVHOLGAVVLTPEOAKAALLDE ILRATQNLDLKKYENLNTEQOKAYEOVOKDLSLLSPETKALLIENH & 160 \\
\hline T. gondi i & GT1. seq & 81 & LIAGQAKAAALQTVHQLGAVALTPEQAKAALLDE ILRATQNLDLRKYENLNTEQQKAYEQVQRDLSQLSPETKALLIENQ & 160 \\
\hline T. gondi i & RH. seq & 161 & RKEKTLLEKARKLFORRHYHVTKOAALAGQILNEORDASGALOSGAVKTAIQRANEOYNVAEEDKNFNEEQHASOLKKVG & 240 \\
\hline T. gond i i & VANDPR. seq & 161 & RKEKGLLEKARKLFORRQYHVGRQAALAGQFLNEQRDSSGALQSGAVKNA INRANEQYNVAEEDKKFNEEQHAAQLKKVG & 240 \\
\hline T. gondi i & ME49. seq & 161 & RKEKSLLEQAKRLFRKRHYHVTRQAALAGQILNEQRDASGALOSGAVKAA IRKANEQYNVAEEDKNFNEEQHAAQLKKVG & 240 \\
\hline T. gondi i & GT1. seq & 161 & RKEKTLLEKARKLFORRHYHVTKOAALAGQ ILNEQRDASGALOSGAVKTAIQRANEQYNVAEEDKNFNEEQHAAQLKKVG & 240 \\
\hline T. gond i i & RH. seq & 241 & AMPSS 245 & \\
\hline T. gondi i & VANDPR. seq & 241 & AMPOS 245 & \\
\hline T. gondi i & ME49. sea & 241 & AMP... 243 & \\
\hline T. gondi i & GT1. seq & 241 & AMPSS 245 & \\
\hline
\end{tabular}

Fig. 1 Alignment of toxofilin protein sequences from toxoplasma strains. Blues letters indicate amino acid differences, while ... represents a missing amino acid 


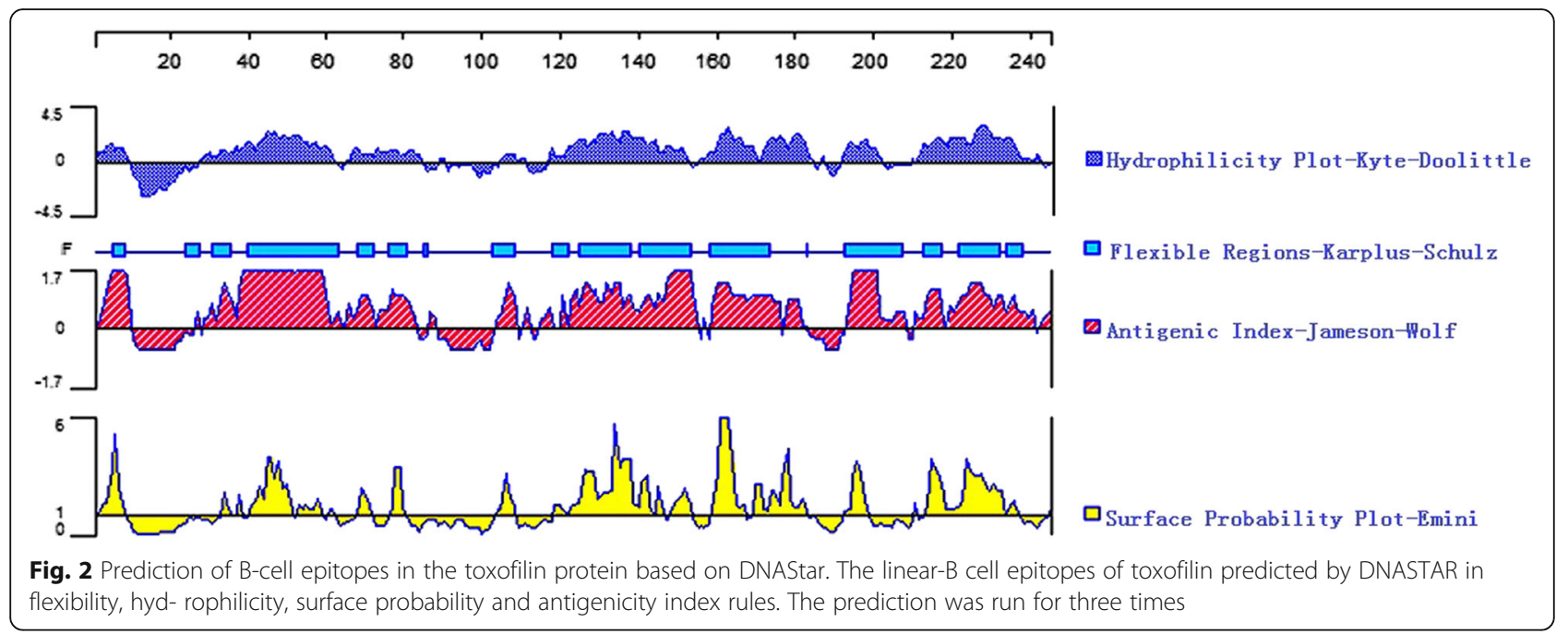

antibody was performed to verify the toxofilin antigen (lane4).

\section{Determination of antibody responses in immunized BALB/c mice}

Sera induced in $T$. gondii immunized mice were tested post-immunization by standard ELISA at weeks $0,2,4$, and 6. As shown in Fig. 5, significantly higher levels of total IgG antibodies were detected in the sera of mice immunized in the experimental groups compared with the control immunized groups, especially after the third immunization. Moreover, the mean titers of IgG antibodies induced in the alum-MPLA- toxofilin group were significantly higher after both priming and booster immunization compared with the levels induced by toxofilin alone DNA vaccine or with single adjuvant. In contrast, the total IgG levels were similar between alumtoxofilin and MPLA-toxofilin groups.

Table 1 Analysis of linear-B cell antigenic epitopes on Tg-toxofilin ${ }^{a}$

\begin{tabular}{llll}
\hline Order & Position & Sequence & Score $^{\mathrm{b}}$ \\
\hline 1 & $5-31$ & KSRPLAAFLLLITVGSLLTASESVQLS & 1.184 \\
2 & $82-105$ & IAGQAKAAALQTVHQLGAVALTPE & 1.153 \\
3 & $107-115$ & AKAALLDEL & 1.089 \\
4 & $175-190$ & QRRHYHVTKQAALAGQ & 1.096 \\
5 & $199-211$ & SGALQSGAVKTAI & 1.095 \\
6 & $233-242$ & SGALQSGAVKTAI & 1.095 \\
7 & $71-78$ & ELGLLRPT & 1.072 \\
8 & $62-69$ & SPSVAARQ & 1.068 \\
9 & $137-158$ & AYEQVQRDLSQLSPETKALLLE & 1.067 \\
\hline
\end{tabular}

${ }^{a}$ The prediction was run for three times. Two or more amino acids condense into a peptide

${ }^{\mathrm{b}}$ High score $=$ high binding
Serum levels of IgG1 and IgG2a, which are characteristic of Th2 and Th1 responses respectively, were analyzed by ELISA after the last immunization. As shown in Fig. 6, the non-adjuvanted toxofilin DNA vaccine group demonstrated similar levels of IgG1 and higher levels of IgG2a compared with control groups. However, compared with the non-adjuvanted toxofilin DNA vaccine, the alum-toxofilin group showed a significant improvement in the IgG1 level, indicative of a Th2-skewed immune response. Conversely, the MPLA-toxofilin group induced higher levels of IgG2a, and is therefore more likely to induce a Th1-biased immune response. Importantly, significantly higher serum levels of IgG2a and a slight increase in IgG1 were detected in the alumMPLA-toxofilin immunized group when compared with other groups, suggesting that this combination of DNA vaccine and adjuvant may be most efficacious in raising a Th1 response following immunization.

\section{Cytokine production by spleen cells}

The sera samples collected were used to measure the levels of IFN- $\gamma, \quad$ IL-4, and IL-10 induced by immunization with toxofilin DNA vaccine in the presence or absence of adjuvants. Generally, IFN- $\gamma$ favors Th1-type immune responses, whereas IL- 4 and IL-10 drive Th2 skewing [16]. As demonstrated in Table 3, values of IFN- $\gamma$ in toxofilin DNA vaccine, alum- toxofilin, and MPLA- toxofilin immunization groups were $607.29 \pm 63.09 \mathrm{pg} / \mathrm{mL}, \quad 694.39 \pm 88.64 \mathrm{pg} / \mathrm{mL}$, and $3043.54 \pm 350.72 \mathrm{pg} / \mathrm{mL}$, respectively, which were significantly higher than in the control group of PBS $(48.54 \pm$ $2.40 \mathrm{pg} / \mathrm{mL})$, alum $(49.58 \pm 2.37 \mathrm{pg} / \mathrm{mL})$, MPLA $(218.34$ $\pm 21.34 \mathrm{pg} / \mathrm{mL})$, alum-MPLA $(267.34 \pm 24.35 \mathrm{pg} / \mathrm{mL})$. Furthermore, the highest level of IFN- $\gamma$ was detected at $3859.42 \pm 380.67 \mathrm{pg} / \mathrm{mL}$ in the alum-MPLA-toxofilin 


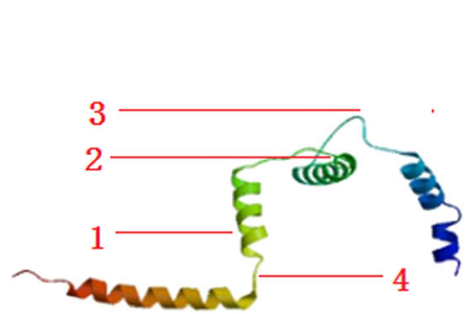

A1

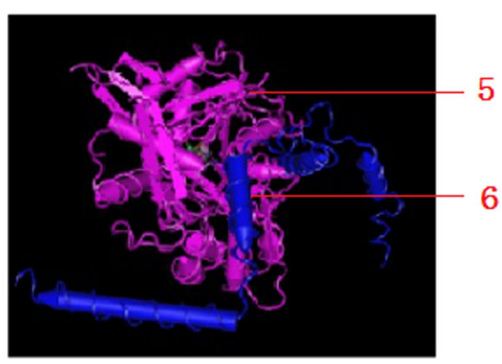

A2

Fig. 3 Toxofilin 3D structure predictions. (A1) 3D structure of Tg-toxofilin 3D model with the highest score for the Tg-toxofilin protein was selected (1: Alpha helix, 2: Random Coil, 3: Extend Strand, 4: Beta Turn). (A2) Complex of Mammalian Actin With Toxofilin From Toxoplasma gondii, Toxofilin from toxoplasma gondii forms a ternary complex with an antiparallel actin dimer. The model was viewed by VMD software and colored to illustrate the different protein components (5: Actin, Alpha skeletal Muscle, 6: toxofilin)

group. Similarly, elevated levels of IL-4 and IL-10 were detected in the alum-toxofilin, MPLA- toxofilin, and alum-MPLA-toxofilin groups. However, significantly higher concentrations of both cytokines (IL-4 236.473 \pm $24.05 \mathrm{pg} / \mathrm{mL}, \mathrm{IL}-10212.32 \pm 30.27 \mathrm{pg} / \mathrm{mL}$ ) were detected in the alum-toxofilin group, compared with the other groups.

\section{T lymphocyte subsets analysis}

The Splenocyte proliferation was evaluated in mice two weeks after the last immunization. As shown in Table 4, $\mathrm{CD} 8+\mathrm{T}$ cells accounted for a significantly higher proportion than that in the control groups and a significant higher percentage of CD8+ T cells were dramatically increased in the alum-MPLA- toxofilin mixture group. The result also showed same statistically difference in the percentage of CD4+ T cells between experimental groups (toxofilin, alum-toxofilin, MPLA-toxofilin and

Table 2 Ligation strength analysis of Tg-toxofilin for MHC class II molecules using SYFPEITHI ${ }^{\mathrm{a}}$

\begin{tabular}{lllc}
\hline MHC II Allele $^{\text {b }}$ & Start-Stop $^{c}$ & Sequence & Percent: e Rank $^{\text {d }}$ \\
\hline HLA-DRB1*01:01 & $91-105$ & LQTVHQLGAVALTPE & 0.39 \\
HLA-DRB1*01:01 & $9-23$ & LAAFLLLIVGSLLT & 0.42 \\
H2-IAb & $78-92$ & EERLIAGQAKAAALQ & 3.04 \\
H2-IAb & $96-110$ & QLGAVALTPEQAKAA & 6.58 \\
H2-IAd & $178-192$ & HYHVTKQAALAGQIL & 0.13 \\
H2-IAd & $231-245$ & QHASQLKKVGAMPSS & 0.62 \\
H2-IEd & $169-183$ & KARKLFQRRHYHVTK & 13.80 \\
H2-IEd & $28-42$ & VQLSEGMKRLSMRGR & 21.98 \\
\hline
\end{tabular}

${ }^{\mathrm{a}}$ The prediction was run for three times

${ }^{\mathrm{b}} \mathrm{H} 2-\mathrm{IAb}, \mathrm{H} 2$-IAd and H2-IEd alleles are mouse MHC class II molecules; the HLADRB 1*01:01 allele is a human MHC class II molecule

'We chose 15 amino acids for analysis each time

${ }^{\mathrm{d}}$ Low percentile $=$ high level binding, high percentile = low level binding alum -MPLA -toxofilin immunization groups) and the control groups.

\section{Protective immunity analysis against challenge with $\mathrm{T}$. gondii RH strain}

The survival curves against acute toxoplasmosis are shown in Fig. 7, and notably prolonged survival times were recorded in the experimentally immunized mice in comparison with the control groups $(P<0.05)$. Among the different experimental groups, mice vaccinated with the alum-MPLA-toxofilin had obviously prolonged survival, with $50 \%$ of mice surviving to day 24 of challenge.

"One month after challenge with 20 cysts of PRU strain of T. gondii, surviving mice were killed and brain cysts were enumerated." The cyst number (1034.49 \pm 45.87) in the mice immunized with alum-MPLAtoxofilin was significantly reduced compared to other groups (Table 5).

\section{Discussion}

Bioinformatics has been regarded as crucial tool in the analysis of the protein characteristics including to the protein epitopes [27]. In this study, bioinformatic analysis has been applied to predict toxofilin antigens using relatedness software and online services. As shown in Fig. 1, the results of our analysis suggested that the toxofilin sequence is conserved between different strains of T. gondii. In addition, the 3D structure of toxofilin was constructed and several promising linear B- and Th-cell epitopes were identified. According to the data analysis, we concluded that toxofilin protein may be regarded as a potential antigen and we have successfully constructed an antigen-encoding plasmid, pEGFP-toxofilin. To further confirm the antigenicity of pEGFP-toxofilin after parasitic infection, the immune response (cellular and humoral) was assessed by immunization and lethal challenge. 


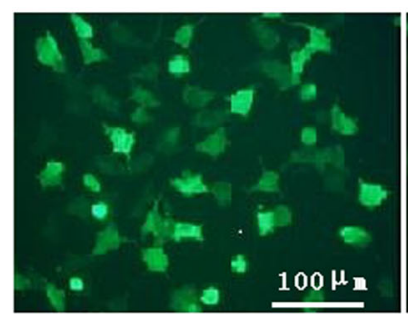

A1

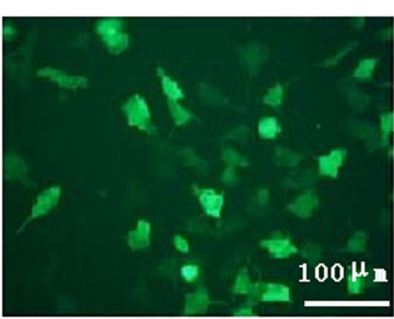

A2

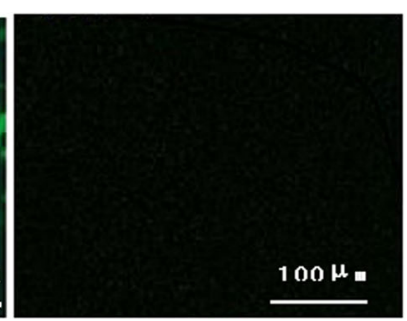

A3

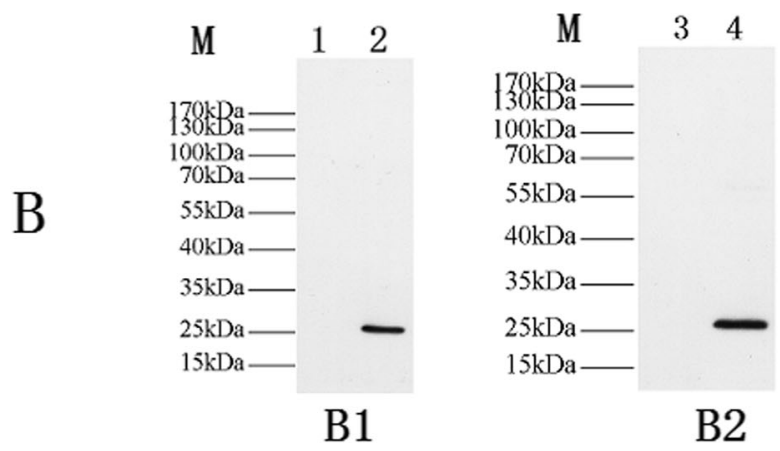

Fig. 4 Green fluorescent microscopy image of HEK293T cells and western blotting. (A1) HEK293 cells transfected with pEGFP-Tgtoxofilin under blue light. (A2) HEK293 cells transfected with pEGFP. (A3) non-transfected HEK293 cells. (B) Western blot analysis of pEGFP-toxofilin protein in transfected HEK293 cells, PET-30 toxofilin protein in bacterial, respectively. (B1) In HEK 293 cells, the expressed proteins were reacted with a T. gondii antibody (lanes 2) and the control empty pEGFP plasmid-transfected cells did not show any band with a T. gondii antibody (lane 1). (B2) PET-30 toxofilin protein were reacted with a T. gondii antibody (lane 4), the empty pET30a vector showed no specific bands with a T. gondii antibody (lane 3). (lane M) Respresents protein molecular weight markers

Generally, infection with $T$. gondii induces a strong and persistent Th1 immune response. IFN- $\gamma$ which is Th1-type cytokine has been regarded as a decisive mediator of resistance to $T$. gondii [28]. In addition, the principal source of IFN- $\gamma$ is from both CD8+ T cells and CD4+ T cells [29]. In this study, the percentage of CD8+

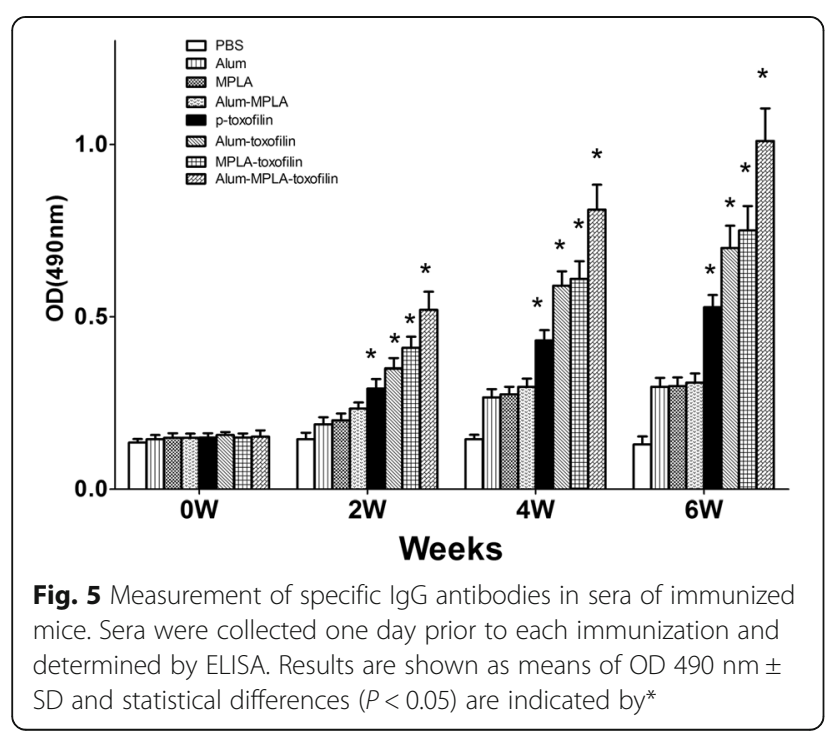

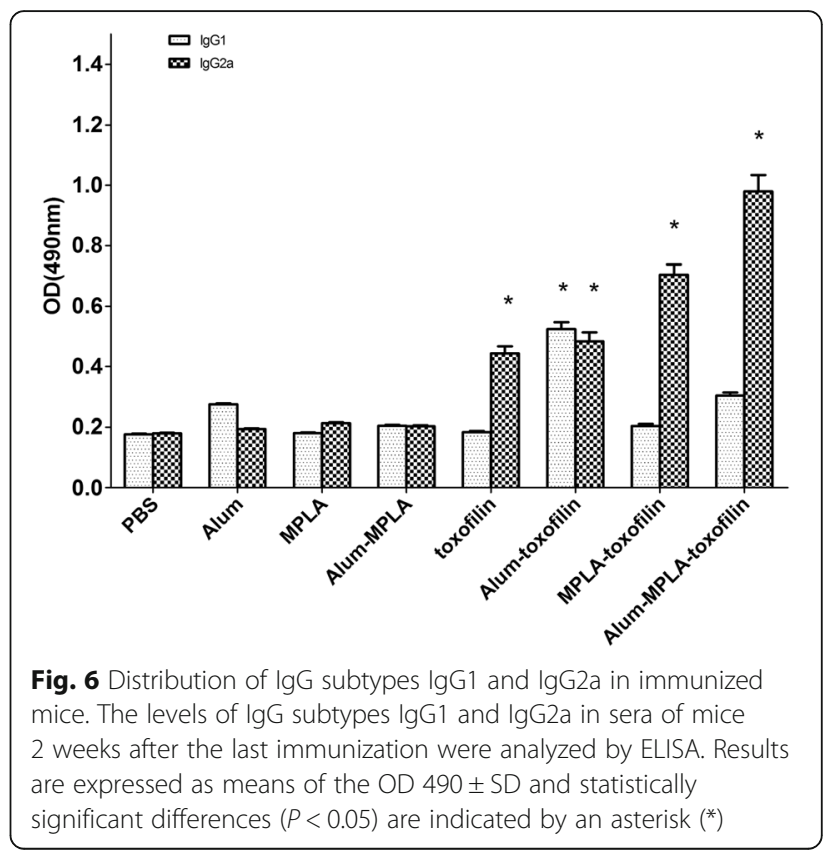


Table 3 Cytokine production by splenocyte ${ }^{a}$ cultures from immunized BALB/c mice

\begin{tabular}{llll}
\hline Groups & \multicolumn{3}{l}{ Cytokine Production $(\mathrm{pg} / \mathrm{ml})^{\mathrm{b}}$} \\
\cline { 2 - 4 } & $\mathrm{IFN}-\mathrm{y}$ & $\mathrm{L}-4$ & $\mathrm{LL}-10$ \\
\hline PBS & $48.54 \pm 2.40$ & $38.79 \pm 2.28$ & $44.70 \pm 2.70$ \\
Alum & $49.58 \pm 2.37$ & $110.49 \pm 10.38$ & $123.45 \pm 13.48$ \\
MPLA & $218.30 \pm 21.34$ & $40.35 \pm 2.34$ & $51.46 \pm 3.21$ \\
alum-MPLA & $267.34 \pm 24.35$ & $78.34 \pm 7.34$ & $58.46 \pm 5.34$ \\
pEGFP- toxofilin & $607.29 \pm 63.09^{*}$ & $37.61 \pm 2.87$ & $34.70 \pm 3.04$ \\
alum-toxofilin & $694.39 \pm 88.64^{*}$ & $236.47 \pm 24.05^{\wedge}$ & $212.32 \pm 30.27^{\wedge}$ \\
MPLA-toxofilin & $3043.54 \pm 350.72^{* \#}$ & $93.58 \pm 10.31$ & $85.43 \pm 9.72$ \\
alum-MPLA- & $3859.42 \pm 380.67^{* \#}$ & $103.43 \pm 24.43$ & $115.32 \pm 30.31$ \\
toxofilin & & &
\end{tabular}

${ }^{a}$ Splenocytes from 3 mice per group two weeks after the final immunization

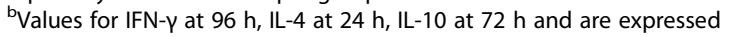
as mean \pm SD

${ }^{*}$ Compared with PBS, alum, MPLA, aum-MPLA, $P<0.05$

\#Compared with alum-toxofilin or pEGFP-toxofilin groups, $P<0.05$

${ }^{\wedge}$ Compared with other groups

$\mathrm{T}$ and $\mathrm{CD} 4+\mathrm{T}$ cells were significantly increased, suggesting that the effect of synergy between CD8+ and CD4+ T cells may contribute to the higher concentration of IFN- $\gamma$. Previous studies have shown IFN- $\gamma$ can also contribute to tryptophan degradation in a large number of infected cell types [29]. As shown in Table 3 and Table 4 , by stimulation with the pEGFP-toxofilin DNA vaccine, a significant increase of IFN- $\gamma, \mathrm{CD} 8+\mathrm{T}$ cells and CD4+ T cells, was induced in comparison with the control groups. Moreover, previous studies have suggested that the level of Th2-associated cytokines, IL-4 and IL-10, produced during T. gondii infection also play an important role in the immune response. In the present study, a slight reduction of both IL-4 and IL-10 was also observed in the pEGFP-toxofilin DNA vaccine

Table 4 Percentages of T lymphocyte subsets in immunized mice ${ }^{a}$ by flow cytometry

\begin{tabular}{lll}
\hline Groups & CD3+ CD4+ CD8- (\%) & CD3+ CD8+ CD4- (\%) \\
\hline PBS & $15.31 \pm 0.12$ & $7.35 \pm 0.18$ \\
alum & $17.34 \pm 0.34$ & $9.62 \pm 0.29$ \\
MPLA & $17.92 \pm 0.25$ & $9.08 \pm 0.24$ \\
alum-MPLA & $18.49 \pm 0.19$ & $10.13 \pm 0.30$ \\
pEGFP- toxofilin & $20.17 \pm 0.53^{*}$ & $12.48 \pm 0.43^{*}$ \\
alum-toxofilin & $21.37 \pm 0.76^{*}$ & $12.98 \pm 0.45^{*}$ \\
MPLA-toxofilin & $27.46 \pm 0.97^{* \#}$ & $17.39 \pm 0.98^{* \#}$ \\
alum-MPLA-toxofiln & $32.38 \pm 1.19^{* \#}$ & $20.68 \pm 1.37^{* \#}$ \\
\hline
\end{tabular}

${ }^{\mathrm{a}}$ Splenocytes from 3 mice per group at the fourth week after the final immunization

${ }^{*}$ Compared with PBS, alum, MPLA, alum-MPLA. $P<0.05$

${ }^{\#}$ Compared with alum-toxofilin or pEGFP-toxofilin groups, $P<0.05$

Results are presented as means \pm SD of three replicate determinations group compared with the other groups, suggesting impaired Th2 responses compared with control groups.

Besides cellular immune responses, humoral immunity resulting in total IgG antibody plays an important role in controlling $T$. gondii infection [30]. In this report, we analyzed the humoral response after vaccination with pEGFP-toxofilin DNA vaccine and found that immunized mice presented high titers of total specific IgG in contrast to the control groups. Along with macrophages, specific IgG antibodies can protect the host from chronic $T$. gondii infections and cyst amount. Our observations were further validated by characterization of the mixed Th1/Th2 response. High IgG1 levels mainly indicate Th2 responses while elevated IgG2a levels favor development of Th1 responses. As shown in Fig. 6, we found higher levels of IgG2a and slightly lower IgG1 titers by the pEGFP-toxofilin DNA vaccine, indicating that our vaccine induced immune responses more biased towards Th1 in BALB/c mice, suggestive of protection against toxoplasmosis and therefore, represents a potential vaccine candidate.

To strengthen and boost the level of molecular vaccines immune responses, adjuvant is a powerful tool to enhance the immunogenicity of DNA vaccines [31]. We evaluated the immunogenicity and protective capacity of pEGFP-toxofilin DNA vaccine either alone, with the single adjuvants alum or MPLA, or combined alum-MPLA adjuvants. Incorporation of alone or mixed adjuvants significantly boosted the production of IgG titers following vaccination with the pEGFP-toxofilin DNA vaccine, inducing differential skewing of protective immunity compared with the non-adjuvanted toxofilin antigen, and to each adjuvant, as alum and MPLA induce different biases in the immune response $[17,18]$. The alumtoxofilin group increased IgG1 levels compared with non-adjuvanted toxofilin, skewing the immune response towards a Th2 response associated with strong production of the IL-4, IL-10, and the IgG1 antibody subtype. Conversely, the MPLA adjuvant is known to induce a Th1-biased immune response [32], resulting in significantly increased specific serum IgG2a isotype antibodies and higher levels of Th1 cytokines. Importantly, the alum-MPLA-toxofilin could effectively lead to a shift from a Th2-skewed immune response towards a Th1 profile and, ultimately, to a balanced immune response compared with the use of a single adjuvant.

For protective efficacy of DNA vaccines against $T$. gondii challenge, the most direct parameter including to survival time, brain cysts load, brain cysts reduction rate, and protection rate should be considered [33]. The present study revealed that the alum-MPLA-toxofilin group could prolong the mice survive time as shown in Fig. 7, significantly reducing the brain cyst burden shown in Table 5. However, our DNA vaccine did not 


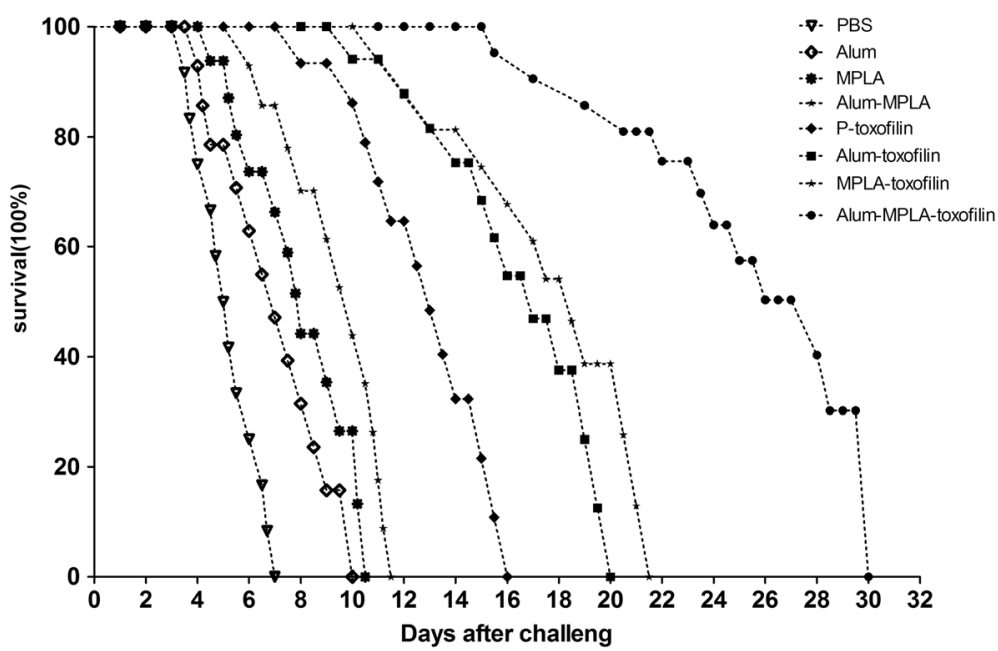

Fig. 7 Survival curves of the vaccinated BALB/C following Toxoplasma gondii challenge infections. The mice in eight groups of mice were challenged with $1 \times 10^{4}$ tachyzoites of the virulent RH strain of T. gondii 2 weeks after the last immunization $(n=12)$ per group

provide complete protection at later time points. In future, evaluating the efficacy of vaccines from different sources remains a new and promising strategy [34]. Further studies are needed to continue to develop DNA vaccines to improve the immunization efficacy against toxoplasmosis.

\section{Conclusions}

In the present study, we use bioinformatic approaches to analyze the antigenicity of $T$. gondii toxofilin antigen. Therefore, toxofilin could be regared as a novel and strong DNA vaccine candidate against toxoplasmosis. At the same time, immunization with the alum-MPLA

Table 5 Mean cyst burden per mouse brain 4 weeks after challenge with 20 cysts of Toxoplasma gondii PRU strain per mouse

\begin{tabular}{ll}
\hline Challenged group $^{a}$ & Brain cysts per mouse $(\text { mean } \pm \text { SD })^{b}$ \\
\hline PBS & $3042.43 \pm 115.17$ \\
alum & $2834.45 \pm 100.89$ \\
MPLA & $2767.36 \pm 103.45$ \\
alum-MPLA & $2532.56 \pm 99.45$ \\
pEGFP-toxofilin & $2048.79 \pm 87.44^{*}$ \\
alum-toxofilin & $1764.17 \pm 78.45^{*}$ \\
MPLA-toxofilin & $1563.42 \pm 80.48^{*}$ \\
alum-MPLA-toxofiln & $1034.49 \pm 45.87^{* \#}$
\end{tabular}

${ }^{a} 10$ mice from each group were challenged intragastrically by 20 cysts 2 weeks after the last immunization. All samples were run for three times ${ }^{\mathrm{b}}$ The mean number of cysts of each group was obtained from every mice brain cysts in the group

"Compared with PBS, alum, MPLA, alum-MPLA, $P<0.05$

${ }^{\#}$ Compared with pEGFP- toxofilin, alum-toxofilin and MPLA-toxofilin, $P<0.05$ mixture as an adjuvant, in combination with the pEGFPtoxofilin DNA vaccine, can enhance cellular immunity and shift the immune response towards a Th1 profile. Furthermore, the alum-MPLA-toxofilin can also induce partial protective immunity in the murine model.

\section{Abbreviations}

DMEM: Dulbecco's modified Eagle's medium; HA: Hemagglutinin; Ig: Immunoglobulin; IL: Interleukin; ip: Intraperitoneally; MHC: Major histocompatibility complex; MPLA: Monophosphoryl lipid A; PBST: PBS containing $0.05 \%$ Tween $20 ;$ TE: Total T. gondii antigen extract; Tg-toxofilin: Toxofilin protein of T. gondii; Th: T helper

\section{Acknowledgments}

We thank all the study participants in China. We would also like to acknowledge Rachel Clausing for her comments on the manuscript.

\section{Funding}

This work was supported, in part, by grants from the National Natural Science Foundation of China (Grant Nos. 81071373,81471974 and 81271857) and the Shandong Provincial Natural Science Foundation (Grant No. ZR2009CM079).

\section{Availability of data and materials}

The amino acid sequences of toxofilin (http://www.ncbi.nlm.nih.gov/genbank), the primary characteristics of the gene encoding toxofilin protein (http:// web.expasy.org/protparam/). T. gondii toxofilin gene [GenBank: AJ132777.2].

\section{Authors'contributions}

$\mathrm{SH}$ and $\mathrm{AZ}$ conceived the idea for the study, and critically revised the manuscript. PS drafted and finalized the manuscript. JZ and GL participated in mouse immunization and challenge. JZ, GL, YH, JG, ZH, HC and HZ performed the experiments. All authors read and approved the final manuscript.

\section{Competing interests}

The authors declare that they have no competing interests.

Consent for publication

Not applicable. 


\section{Ethics approval and consent to participate}

The Institutional Animal Care and Use Committee of Shandong University approved all experimental protocols. Experiments were performed in accordance with the criteria for the care and use of laboratory animals.

\section{Author details}

'Department of Parasitology, Shandong University School of Medicine, Jinan, Shandong Province 250012, People's Republic of China. ${ }^{2}$ Shandong University School of Medicine, 250021 Jinan, Shandong Province, People's Republic of China. ${ }^{3}$ Department of Pediatrics, Provincial Hospital Affiliated to Shandong University, Shandong University School of Medicine, 250021 Jinan, Shandong Province, People's Republic of China.

Received: 23 August 2016 Accepted: 21 December 2016 Published online: 05 January 2017

\section{References}

1. Dubey JP. The history of Toxoplasma gondii-the first 100 years. J Eukaryot Microbiol. 2008;55(6):467-75.

2. Swierzy IJ, Muhammad M, Kroll J, Abelmann A, Tenter AM, Luder CG. Toxoplasma gondii within skeletal muscle cells: a critical interplay for foodborne parasite transmission. Int J Parasitol. 2014;44(2):91-8.

3. Tenter AM, Heckeroth AR, Weiss LM. Toxoplasma gondii: from animals to humans. Int J Parasitol. 2000;30(12-13):1217-58.

4. Yang N, Mu MY, Li HK, Long M, He JB. Seroprevalence of Toxoplasma gondii infection in slaughtered chickens, ducks, and geese in Shenyang, northeastern China. Parasit Vectors. 2012;5:237.

5. Cenci-Goga BT, Rossitto PV, Sechi P, McCrindle CM, Cullor JS. Toxoplasma in animals, food, and humans: an old parasite of new concern. Foodborne Pathog Dis. 2011;8(7):751-62.

6. Meng M, Zhou A, Lu G, Wang L, Zhao G, Han Y, Zhou H, Cong H, Zhao Q, Zhu $X Q$, et al. DNA prime and peptide boost immunization protocol encoding the Toxoplasma gondii GRA4 induces strong protective immunity in BALB/c mice. BMC Infect Dis. 2013;13:494.

7. Kur J, Holec-Gasior L, Hiszczynska-Sawicka E. Current status of toxoplasmosis vaccine development. Expert Rev Vaccines. 2009;8(6):791-808.

8. Zhang NZ, Huang SY, Xu Y, Chen J, Wang JL, Tian WP, Zhu XQ. Evaluation of immune responses in mice after DNA immunization with putative Toxoplasma gondii calcium-dependent protein kinase 5. Clin Vaccine Immunol. 2014;21(7):924-9.

9. Gong P, Huang X, Yu Q, Li Y, Huang J, Li J, Yang J, Li H, Zhang G, Ren W, et al. The protective effect of a DNA vaccine encoding the Toxoplasma gondii cyclophilin gene in BALB/C mice. Parasite Immunol. 2013;35(3-4):140-6.

10. Parthasarathy S, Fong MY, Ramaswamy K, Lau YL. Protective immune response in BALB/C mice induced by DNA vaccine of the ROP8 gene of Toxoplasma gondii. Am J Trop Med Hyg. 2013;88(5):883-7.

11. Sun XM, Zou J, Elashram Saeed AA, Yan WC, Liu XY, Suo X, Wang H, Chen QJ. DNA vaccination with a gene encoding Toxoplasma gondii GRA6 induces partial protection against toxoplasmosis in BALB/C mice. Parasit Vectors. 2011;4:213.

12. Lee SH, Hayes DB, Rebowski G, Tardieux I, Dominguez R. Toxofilin from Toxoplasma gondii forms a ternary complex with an antiparallel actin dimer. Proc Natl Acad Sci U S A. 2007:104(41):16122-7.

13. Lodoen MB, Gerke C, Boothroyd JC. A highly sensitive FRET-based approach reveals secretion of the actin-binding protein toxofilin during Toxoplasma gondii infection. Cell Microbiol. 2010;12(1):55-66.

14. Baz M, Samant M, Zekki H, Tribout-Jover P, Plante M, Lanteigne AM, Hamelin ME, Mallett C, Papadopoulou B, Boivin G. Effects of different adjuvants in the context of intramuscular and intranasal routes on humoral and cellular immune responses induced by detergent-split A/H3N2 influenza vaccines in mice. Clin Vaccine Immunol. 2012;19(2):209-18.

15. Singh M, Srivastava I. Advances in vaccine adjuvants for infectious diseases. Curr HIV Res. 2003;1(3):309-20.

16. Azmi F, Ahmad FA, Skwarczynski M, Toth I. Recent progress in adjuvant discovery for peptide-based subunit vaccines. Hum Vaccin Immunother. 2014;10(3):778-96.

17. Hu X, Liu R, Zhu N. Enhancement of humoral and cellular immune responses by monophosphoryl lipid A (MPLA) as an adjuvant to the rabies vaccine in BALB/C mice. Immunobiology. 2013;218(12):1524-8.
18. Nouri A, Laraba-Djebari F. Enhancement of long-lasting immunoprotective effect against Androctonus australis hector envenomation using safe antigens: Comparative role of MF59 and Alum adjuvants. Vaccine. 2015:33(43):5756-63.

19. Alving CR, Peachman KK, Rao M, Reed SG. Adjuvants for human vaccines. Curr Opin Immunol. 2012;24(3):310-5.

20. Alving CR, Rao M, Steers NJ, Matyas GR, Mayorov AV. Liposomes containing lipid $A$ : an effective, safe, generic adjuvant system for synthetic vaccines. Expert Rev Vaccines. 2012;11(6):733-44.

21. Greenbaum JA, Andersen PH, Blythe M, Bui HH, Cachau RE, Crowe J, Davies M, Kolaskar AS, Lund O, Morrison S, et al. Towards a consensus on datasets and evaluation metrics for developing B-cell epitope prediction tools. J Mol Recognit. 2007;20(2):75-82

22. Sun P, Ju H, Liu Z, Ning Q, Zhang J, Zhao X, Huang Y, Ma Z, Li Y. Bioinformatics resources and tools for conformational B-cell epitope prediction. Comput Math Methods Med. 2013;2013:943636.

23. Yao B, Zheng D, Liang S, Zhang C. Conformational B-cell epitope prediction on antigen protein structures: a review of current algorithms and comparison with common binding site prediction methods. PLoS One. 2013;8(4):e62249.

24. Wang Y, Wang G, Zhang D, Yin H, Wang M. Identification of novel B cell epitopes within Toxoplasma gondii GRA1. Exp Parasitol. 2013;135(3):606-10.

25. Zhao G, Zhou A, Lu G, Meng M, Sun M, Bai Y, Han Y, Wang L, Zhou H, Cong $\mathrm{H}$, et al. Identification and characterization of Toxoplasma gondii aspartic protease 1 as a novel vaccine candidate against toxoplasmosis. Parasit Vectors. 2013:6:175.

26. Yu L, Yamagishi J, Zhang S, Jin C, Aboge GO, Zhang H, Zhang G, Tanaka T, Fujisaki K, Nishikawa Y, et al. Protective effect of a prime-boost strategy with plasmid DNA followed by recombinant adenovirus expressing TgAMA1 as vaccines against Toxoplasma gondii infection in mice. Parasitol Int. 2012:61(3):481-6.

27. Golkar M, Shokrgozar MA, Rafati S, Musset K, Assmar M, Sadaie R, CesbronDelauw MF, Mercier C. Evaluation of protective effect of recombinant dense granule antigens GRA2 and GRA6 formulated in monophosphoryl lipid A (MPL) adjuvant against Toxoplasma chronic infection in mice. Vaccine. 2007;25(21):4301-11.

28. Sharma P, Gaur SN, Arora N. In silico identification of IgE-binding epitopes of osmotin protein. PLoS One. 2013;8(1):e54755.

29. Suzuki Y, Orellana MA, Schreiber RD, Remington JS. Interferon-gamma: the major mediator of resistance against Toxoplasma gondii. Science. 1988;240(4851):516-8.

30. Dawson HD, Beshah E, Nishi S, Solano-Aguilar G, Morimoto M, Zhao A, Madden KB, Ledbetter TK, Dubey JP, Shea-Donohue T, et al. Localized multigene expression patterns support an evolving Th1/Th2-like paradigm in response to infections with Toxoplasma gondii and Ascaris suum. Infect Immun. 2005;73(2):1116-28.

31. Johnson LL, Lanthier P, Hoffman J, Chen W. Vaccination protects B celldeficient mice against an oral challenge with mildly virulent Toxoplasma gondii. Vaccine. 2004;22(29-30):4054-61.

32. Schulke $S$, Vogel $L$, Junker AC, Hanschmann KM, Flaczyk A, Vieths $S$, Scheurer S. A Fusion Protein Consisting of the Vaccine Adjuvant Monophosphoryl Lipid A and the Allergen Ovalbumin Boosts AllergenSpecific Th1, Th2, and Th17 Responses In Vitro. J Immunol Res. 2016;2016:4156456.

33. Li J, Huang $X$, Zhang G, Gong P, Zhang $X$, Wu L. Immune response and protective efficacy against homologous challenge in BALB/c mice vaccinated with DNA vaccine encoding Toxoplasma gondii actin depolymerizing factor gene. Vet Parasitol. 2011;179(1-3):1-6.

34. Jongert E, Roberts CW, Gargano N, Forster-Waldl E, Petersen E. Vaccines against Toxoplasma gondii: challenges and opportunities. Mem Inst Oswaldo Cruz. 2009:104(2):252-66. 